\title{
Revisiting the Publication Culture in Computing Research
}

\author{
In my May 2009 Editor”s Letter, "Conferences vs. Journals \\ in Computing Research" (p. 5), I addressed the publication \\ culture of our field: "As far as I know, we are the only \\ scientific community that considers conference publication
}

as the primary means of publishing our research results. In contrast, the prevailing academic standard of 'publish' is 'publish in archival journals.' Why are we the only discipline driving on the conference side of the "publication road?"”

In response to my editorial, Lance Fortnow wrote a Viewpoint column (Aug. 2009, p. 33), entitled "Time for Computer Science to Grow Up," in which he concluded: "Computer science has grown to become a mature field where no major university can survive without a strong CS department. It is time for computer science to grow up and publish in a way that represents the major discipline it has become."

The May 2009 editorial and the August 2009 column attracted a lot of attention in the blogosphere. The reaction has been mostly sympathetic to the point of view reflected in both pieces. For example, Jeanette Wing asked in her blog: "How can we break the cycle of deadline-driven research?", and Filippo Menczer, in a Letter to the Editor published in the November 2009 issue, said: "I propose the abolition of conference proceedings altogether."

Not everyone, however, agreed with this point of view. For example, in another Letter to the Editor from the November 2009 issue, Jano van Hemert said: "For CS to grow up, CS journals must grow up first." Mr. van Hemert's issue with computing-research journals is that they are known to have "slow turnaround, with most taking at least a year to make a publish/reject decision and some taking much longer before publishing." Such end-to-end times, he argued, "are unheard of in other fields where journal editors make decisions in weeks, sometimes days."

While I have not see concrete data comparing publishing turnaround times for computing-research journals to those in other technical fields, there is abundance of anecdotal data supporting the claim that computingresearch journals are indeed quite slow. (The average time to editorial decision for Communications is under two months; that takes a concerted effort by the editorial board to ensure that the editorial process does not stall.)

What is the reason for the unacceptably slow turnaround time in computing-research journals? When considering this question, one must factor the problem into two separate issues: time from submission to editorial decision, and time from positive editorial decision to publication.

First let us address the latter issue. All periodical journals have editorial "pipelines." No publisher wants to face the threat of an empty issue; it's akin to the dreaded dead air on television! Successful journals that attract many submissions often see their pipelines extend for up to two years. With the advent of electronic publishing, this problem can be eliminated or at least minimized. Communications uses its Virtual Extension (VE) to ensure its pipeline does not get longer than six months. VE articles undergo the same rigorous review process as those in the print edition and are accepted for publication on their merit. These articles are available in ACM's Digital Library.

Let us now consider the editorial process in computing-research journals. Why is it soooo slow? Consider who is in charge of that process. It is not the publishers; it is the editors and referees. In other words, it is us. The process is slow because that is the way we run it. If we want it changed, it is up to us to change it! I suspect that we cannot separate our conference-focused publication culture from our sluggish journal editorial process. Conferences have sharp deadlines, journals do not. We simply do not take our roles as editors and referees as seriously as we do as program committee members because we do not take journals as seriously as other fields. If we, as a community, decide that we need to shift from conference-based publication to journal-based publication, we definitely must address the slow editorial process, but we should not complain about "them journals." We have found the enemy, and it is us!

The 2010 Conference of the Computing Research Association (July 1820, Snowbird, UT) will have a plenary panel on "Peer Review in Computing Research." I look forward to that discussion and hope it will help our community reach consensus on this issue.

Moshe Y. Vardi, EDITOR-IN-CHIEF 